\title{
Multi-Hamiltonian Structures on Spaces of Closed Equicentroaffine Plane Curves Associated to Higher KdV Flows
}

\author{
Atsushi FUJIOKA ${ }^{\dagger}$ and Takashi KUROSE ${ }^{\ddagger}$ \\ $\dagger$ Department of Mathematics, Kansai University, Suita, 564-8680, Japan \\ E-mail: afujioka@kansai-u.ac.jp \\ $\ddagger$ Department of Mathematical Sciences, Kwansei Gakuin University, Sanda, 669-1337, Japan \\ E-mail: crg31562@kwansei.ac.jp
}

Received October 11, 2013, in final form April 16, 2014; Published online April 22, 2014

http://dx.doi.org/10.3842/SIGMA.2014.048

\begin{abstract}
Higher KdV flows on spaces of closed equicentroaffine plane curves are studied and it is shown that the flows are described as certain multi-Hamiltonian systems on the spaces. Multi-Hamiltonian systems describing higher mKdV flows are also given on spaces of closed Euclidean plane curves via the geometric Miura transformation.
\end{abstract}

Key words: motions of curves; equicentroaffine curves; KdV hierarchy; multi-Hamiltonian systems

2010 Mathematics Subject Classification: 37K25; 35Q53

\section{Introduction}

A motion of a curve is a smooth one-parameter family of connected curves in a space. It is known that many differential equations related to integrable systems can be linked with special motions of curves [10, 11, 12, 29]. For example, for a special motion of an inextensible curve in the Euclidean plane, the curvature evolves according to the modified Korteweg-de Vries (mKdV) equation [19] (cf. Section 4 below). There are a lot of preceding studies on motions of curves related to Euclidean geometry and the $\mathrm{mKdV}$ equation. See [24, 30, 32] and references therein. For special motions of a space curve, it is also known that the nonlinear Schrödinger equation appears [16]. In $[13,14]$, the authors studied motions of a curve in the complex hyperbola under which the curvature evolves according to the Burgers equation.

In this paper, we shall study motions of an equicentroaffine plane curve. Under a special motion of an equicentroaffine plane curve, the equicentroaffine curvature evolves according to the Korteweg-de Vries (KdV) equation. In order to explain the above motion geometrically, Pinkall [28] introduced the natural presymplectic form on the space of closed equicentroaffine plane curves with fixed enclosing area, and showed that the equicentroaffine curvature evolves according to the $\mathrm{KdV}$ equation when the flow is generated by the total equicentroaffine curvature. Furthermore, the result has been generalized to the case of higher KdV flows (cf. [9, 15]).

On the other hand, it is known that a lot of completely integrable systems are described as biHamiltonian systems, from which the existence of many first integrals can be deduced (Magri's theorem [22, 27]). In this context, many of motions of curves as above have been studied from the viewpoint of bi-Hamiltonian systems recently $[1,2,3,4,5,6,7,8,21,23,24,31]$. The purpose of this paper is to construct a multi-Hamiltonian structure associated to the higher KdV flows on each level set of Hamiltonian functions in a geometric way (Theorem 7). Moreover, we shall also introduce multi-Hamiltonian structures associated to the higher $\mathrm{mKdV}$ flows on the spaces of closed Euclidean plane curves via the geometric Miura transformation. 


\section{A bi-Hamiltonian structure on the space of closed equicentroaffine curves}

Throughout this paper all maps are assumed to be smooth.

For a regular plane curve $\gamma$ whose velocity vector is transversal to the position vector at each point, we can choose the parameter $s$ of $\gamma$ as $\operatorname{det}\left(\begin{array}{c}\gamma(s) \\ \gamma_{s}(s)\end{array}\right) \equiv 1$ holds. A plane curve $\gamma$ provided with such a parameter $s$ is called an equicentroaffine plane curve. For an equicentroaffine plane curve $\gamma$, we can define a function $\kappa$, called the equicentroaffine curvature, by $\gamma_{s s}=-\kappa \gamma$.

We set the space $\mathcal{M}$ of closed equicentroaffine plane curves by

$$
\mathcal{M}=\left\{\gamma: S^{1} \rightarrow \mathbb{R}^{2} \backslash\{0\} \mid \operatorname{det}\left(\begin{array}{c}
\gamma \\
\gamma_{s}
\end{array}\right)=1\right\}
$$

where $S^{1}=\mathbb{R} / 2 \pi \mathbb{Z}$. Let $\gamma(\cdot, t) \in \mathcal{M}$ be a one-parameter family of closed equicentroaffine plane curves. As in [28], the motion vector field $\gamma_{t}$ is represented as

$$
\gamma_{t}=-\frac{1}{2} \alpha_{s} \gamma+\alpha \gamma_{s}, \quad \alpha: S^{1} \rightarrow \mathbb{R},
$$

and the equicentroaffine curvature $\kappa$ evolves as

$$
\kappa_{t}=\Omega \alpha_{s}=\frac{1}{2} \alpha_{s s s}+2 \kappa \alpha_{s}+\kappa_{s} \alpha,
$$

where

$$
\Omega=\frac{1}{2} D_{s}^{2}+2 \kappa+\kappa_{s} D_{s}^{-1}, \quad D_{s}=\frac{\partial}{\partial s},
$$

is the recursion operator of the KdV equation:

$$
\kappa_{t}=\Omega \kappa_{s}=\frac{1}{2} \kappa_{s s s}+3 \kappa \kappa_{s} .
$$

Hence when we choose the one-parameter family $\gamma(\cdot, t)$ as $\alpha=D_{s}^{-1} \Omega^{n-1} \kappa_{s}$, we obtain the $n$th $\mathrm{KdV}$ equation for $\kappa$ :

$$
\kappa_{t}=\Omega^{n} \kappa_{s} .
$$

The tangent space of $\mathcal{M}$ at $\gamma \in \mathcal{M}$ is described as

$$
T_{\gamma} \mathcal{M}=\left\{-\frac{1}{2} \alpha_{s} \gamma+\alpha \gamma_{s} \mid \alpha: S^{1} \rightarrow \mathbb{R}\right\},
$$

and we can define a presymplectic form $\omega_{0}$ on $\mathcal{M}$ by

$$
\omega_{0}(X, Y)=\int_{S^{1}} \operatorname{det}\left(\begin{array}{l}
X \\
Y
\end{array}\right) d s, \quad X, Y \in T_{\gamma} \mathcal{M} .
$$

When $X$ and $Y$ are given by

$$
X=-\frac{1}{2} \alpha_{s} \gamma+\alpha \gamma_{s}, \quad Y=-\frac{1}{2} \beta_{s} \gamma+\beta \gamma_{s}, \quad \alpha, \beta: S^{1} \rightarrow \mathbb{R},
$$

a direct calculation shows that

$$
\omega_{0}(X, Y)=\int_{S^{1}} \alpha \beta_{s} d s,
$$

from which we see that the kernel of $\omega_{0}$ at $\gamma$ is $\mathbb{R} \cdot \gamma_{s}$. 
It is known that the higher KdV equation (3) as well as (2) has an infinite series of conserved quantities $\left\{H_{m}\right\}_{m \in \mathbb{N}}$ given in the form of

$$
H_{m}=\int_{S^{1}} h_{m}\left(\kappa, \kappa_{s}, \kappa_{s s}, \ldots\right) d s
$$

where $h_{m}$ is a polynomial in $\kappa$ and its derivatives up to order $m$, for example,

$$
h_{1}=\kappa, \quad h_{2}=\frac{1}{2} \kappa^{2}, \quad h_{3}=\frac{1}{2} \kappa^{3}-\frac{1}{4} \kappa_{s}^{2}
$$

(see [17, 20, 25, 26]). Moreover, by using the conserved quantity, $n$th KdV equation (3) can be expressed as

$$
\kappa_{t}=D_{s} \frac{\delta H_{n+2}}{\delta \kappa}
$$

where $\delta H_{n+2} / \delta \kappa$ is the variational derivative of $H_{n+2}$ :

$$
\frac{\delta H_{n+2}}{\delta \kappa}=\frac{\partial h_{n+2}}{\partial \kappa}-D_{s} \frac{\partial h_{n+2}}{\partial \kappa_{s}}+D_{s}^{2} \frac{\partial h_{n+2}}{\partial \kappa_{s s}}-\cdots .
$$

The expression (5) played an important role in computation in [15], where we studied the higher $\mathrm{KdV}$ flows on the space of closed equicentroaffine curves as Hamiltonian systems; using the above presymplectic structure $\omega_{0}$, we gave the Hamiltonian flows associated with the higher KdV equations. The paper [15] deals also with the geometric Miura transformation as is mentioned in Section 5 below.

For each $n \in \mathbb{N}$, we define a vector field $X_{n}$ on $\mathcal{M}$ by

$$
\left(X_{n}\right)_{\gamma}=-\frac{1}{2}\left(\Omega^{n-1} \kappa_{s}\right) \gamma+\left(D_{s}^{-1} \Omega^{n-1} \kappa_{s}\right) \gamma_{s}, \quad \gamma \in \mathcal{M}
$$

Regarding $\left\{H_{m}\right\}_{m \in \mathbb{N}}$ as functions on $\mathcal{M}$ by substituting the equicentroaffine curvature of $\gamma$ for $\kappa$, we have the following proposition, which is essentially due to Pinkall [28] in the case $n=1$.

Proposition 1 ([15]). For each $n \in \mathbb{N}, X_{n}$ is a Hamiltonian vector field for $H_{n}$ with respect to $\omega_{0}$, i.e., $d H_{n}=\omega_{0}\left(X_{n}, \cdot\right)$ holds. Hence $H_{n}$ is a Hamiltonian function for the nth KdV flow $\gamma_{t}=X_{n}$.

Now, we define another form $\omega_{1}$ on $\mathcal{M}$ by

$$
\omega_{1}(X, Y)=\int_{S^{1}} \operatorname{det}\left(\begin{array}{c}
X \\
\left(D_{s}^{2}+\kappa\right) Y
\end{array}\right) d s, \quad X, Y \in T_{\gamma} \mathcal{M},
$$

which is represented as

$$
\omega_{1}(X, Y)=\int_{S^{1}} \alpha \Omega \beta_{s} d s
$$

for $X, Y$ given by (4). The following shows that $\omega_{0}$ and $\omega_{1}$ with $\left\{H_{m}\right\}_{m \in N}$ define a biHamiltonian structure on $\mathcal{M}$ (cf. [22, 27]).

Theorem 2. The form $\omega_{1}$ is a presymplectic form on $\mathcal{M}$. For each $n \in \mathbb{N}, X_{n}$ is a Hamiltonian vector field for $H_{n+1}$ with respect to $\omega_{1}$. 
Proof. For two functions $F$ and $G$ on $\mathcal{M}$ of the form

$$
F=\int_{S^{1}} f\left(\kappa, \kappa_{s}, \kappa_{s s}, \ldots\right) d s, \quad G=\int_{S^{1}} g\left(\kappa, \kappa_{s}, \kappa_{s s}, \ldots\right) d s,
$$

we set

$$
\{F, G\}_{1}=\int_{S^{1}} \frac{\delta F}{\delta \kappa} \Omega D_{s} \frac{\delta G}{\delta \kappa} d s .
$$

Then from $[18,22]$, we see that $\{\cdot, \cdot\}_{1}$ provides a Poisson bracket with

$$
X_{n}=-\left\{H_{n+1}, \cdot\right\}_{1} \text {. }
$$

We put $\widetilde{\alpha}_{F}=\delta F / \delta \kappa$ and $\left(\widetilde{X}_{F}\right)_{\gamma}=-(1 / 2)\left(\widetilde{\alpha}_{F}\right)_{s} \gamma+\widetilde{\alpha}_{F} \gamma_{s}$. Since the differentiation of $F$ along a motion $\gamma_{t}=X_{\gamma}=-(1 / 2) \alpha_{s} \gamma+\alpha \gamma_{s}$ is given as

$$
X F=\frac{d F}{d t}=\int_{S^{1}} \frac{\delta F}{\delta \kappa} \kappa_{t} d s=\int_{S^{1}} \frac{\delta F}{\delta \kappa} \Omega \alpha_{s} d s,
$$

we have

$$
\omega_{1}\left(\widetilde{X}_{F}, X\right)=\int_{S^{1}} \frac{\delta F}{\delta \kappa} \Omega \alpha_{s} d s=X F=d F(X)
$$

and

$$
\omega_{1}\left(\widetilde{X}_{F}, \widetilde{X}_{G}\right)=\int_{S^{1}} \frac{\delta F}{\delta \kappa} \Omega D_{s} \frac{\delta G}{\delta \kappa}=\{F, G\}_{1} .
$$

Hence $\omega_{1}$ is skew-symmetric and its closedness follows from the Jacobi identity for $\{\cdot, \cdot\}_{1}$ since for functions $F, G$ and $H=\int_{S^{1}} h\left(\kappa, \kappa_{s}, \kappa_{s s}, \ldots\right) d s$ on $\mathcal{M}$ we have

$$
d \omega\left(\widetilde{X}_{F}, \widetilde{X}_{G}, \widetilde{X}_{H}\right)=2\left(\left\{\{F, G\}_{1}, H\right\}_{1}+\left\{\{G, H\}_{1}, F\right\}_{1}+\left\{\{H, F\}_{1}, G\right\}_{1}\right)=0 .
$$

Moreover, since

$$
\widetilde{X}_{F} G=\int_{S^{1}} \frac{\delta G}{\delta \kappa} \Omega D_{s} \frac{\delta F}{\delta \kappa} d s=\{G, F\}_{1}=-\{F, G\}_{1},
$$

we obtain $X_{n}=\widetilde{X}_{H_{n+1}}$ and hence

$$
\omega_{1}\left(X_{n}, \cdot\right)=\omega_{1}\left(\tilde{X}_{H_{n+1}}, \cdot\right)=d H_{n+1} \cdot
$$

Therefore $X_{n}$ is a Hamiltonian vector field for $H_{n+1}$ with respect to $\omega_{1}$.

The special linear group of degree two $\operatorname{SL}(2 ; \mathbb{R})$ acts on $\mathcal{M}$ as $\mathcal{M} \ni \gamma \mapsto A \gamma \in \mathcal{M}(A \in$ $\mathrm{SL}(2 ; \mathbb{R}))$. Two elements of $\mathcal{M}$ belong to the same orbit if and only if their equicentroaffine curvatures coincide. Hence $\omega_{1}$ is invariant under the action of $\operatorname{SL}(2 ; \mathbb{R})$. Moreover, the kernel of $\omega_{1}$ at $\gamma$ is the tangent space of the orbit $\mathrm{SL}(2 ; \mathbb{R}) \cdot \gamma$; indeed for a one-parameter family $\gamma(\cdot, t) \in \mathcal{M}$, it follows from (2) and (6) that the tangent vector (1) belongs to the kernel of $\omega_{1}$ if and only if $\kappa_{t}=0$, that is, $\kappa$ is independent of $t$ and hence $\gamma(\cdot, t)$ is contained in an $\mathrm{SL}(2 ; \mathbb{R})$-orbit. As a consequence, $\omega_{1}$ defines a symplectic form on the quotient space $\mathcal{M} / \mathrm{SL}(2 ; \mathbb{R})$.

We consider another action on $\mathcal{M}$ given by

$$
\mathcal{M} \ni \gamma \mapsto \gamma(\cdot+\sigma) \in \mathcal{M}, \quad \sigma \in S^{1} .
$$

It is obvious that this $S^{1}$-action is presymplectic, that is, it leaves $\omega_{1}$ invariant. Moreover, the action is Hamiltonian as we see in the proof of the following theorem. 
Theorem 3. The moment map $\mu_{1}$ for the $S^{1}$-action (8) with respect to $\omega_{1}$ is given by

$$
\mu_{1}(\gamma)\left(\frac{\partial}{\partial \sigma}\right)=H_{1}(\gamma), \quad \gamma \in \mathcal{M}
$$

Proof. The fundamental vector field $\underline{A}$ on $\mathcal{M}$ corresponding to $\partial / \partial \sigma \in \operatorname{Lie}\left(S^{1}\right)$ is given by $\underline{A}_{\gamma}=\gamma_{s}(\gamma \in \mathcal{M})$. For any tangent vector $\gamma_{t}=-(1 / 2) \alpha_{s} \gamma+\alpha \gamma_{s}$, we have

$$
\omega_{1}\left(\underline{A}, \gamma_{t}\right)=\omega_{1}\left(\gamma_{s}, \gamma_{t}\right)=\int_{S^{1}} \Omega \alpha_{s} d s=\int_{S^{1}} \kappa_{t} d s=\frac{d}{d t} H_{1}(\gamma)=d H_{1}\left(\gamma_{t}\right)
$$

which implies (9) by the definition of the moment map.

Remark 4. Let $\Phi_{n}^{\tau}$ be the flow generated by $X_{n}$, that is, $\Phi_{n}$ is a one-parameter transformation group of $\mathcal{M}$ such that

$$
\left.\frac{\partial}{\partial \tau}\right|_{\tau=0} \Phi_{n}^{\tau}(\gamma)=\left(X_{n}\right)_{\gamma}, \quad \gamma \in \mathcal{M}
$$

As an $\mathbb{R}$-action on $\mathcal{M}, \Phi_{n}$ is Hamiltonian with respect to $\omega_{0}$ and the corresponding moment map is given by $H_{n}$.

\section{Multi-Hamiltonian structures on the level sets of Hamiltonians}

For a given sequence of real numbers $C=\left\{c_{k}\right\}_{k \in \mathbb{N}}$, we define subsets $\mathcal{M}\left(C_{m}\right)(m=1,2, \ldots)$ of $\mathcal{M}$ by

$$
\mathcal{M}\left(C_{m}\right)=H_{1}^{-1}\left(c_{1}\right) \cap \cdots \cap H_{m}^{-1}\left(c_{m}\right) .
$$

In the following, we assume that each $\mathcal{M}\left(C_{m}\right)$ is not an empty set.

Lemma 5. For functions $\alpha, \beta$ on $S^{1}$, if $D_{s}^{-1} \Omega D_{s} \alpha$ is determined as a function on $S^{1}$, then we have

$$
\int_{S^{1}}\left(D_{s}^{-1} \Omega D_{s} \alpha\right) \cdot \beta_{s} d s=\int_{S^{1}} \alpha \Omega \beta_{s} d s
$$

Proof. Noting $\Omega D_{s}=(1 / 2) D_{s}^{3}+\kappa D_{s}+D_{s} \kappa$, we can easily verify (10) by integration by parts.

Proposition 6. For $\gamma \in \mathcal{M}\left(C_{m}\right)$ and $X=-(1 / 2) \alpha_{s} \gamma+\alpha \gamma_{s} \in T_{\gamma} \mathcal{M}\left(C_{m}\right), \Omega \alpha_{s}, \Omega^{2} \alpha_{s}, \ldots$, $\Omega^{m+1} \alpha_{s}$ are defined as functions on $S^{1}$ and $\int_{S^{1}} \Omega^{k} \alpha_{s} d s=0$ for any $k=1,2, \ldots, m$.

Proof. We shall show the proposition by induction on $m$. In the case $m=1, \Omega \alpha_{s}=(1 / 2) \alpha_{s s s}+$ $2 \kappa \alpha_{s}+\kappa_{s} \alpha$ is a function on $S^{1}$ and we have

$$
\int_{S^{1}} \Omega \alpha_{s} d s=\int_{S^{1}} \kappa \alpha_{s} d s=\omega_{0}\left(X_{1}, X\right)=d H_{1}(X)
$$

which vanishes since $X \in T_{\gamma} \mathcal{M}\left(C_{1}\right)=\operatorname{Ker}\left(d H_{1}\right)_{\gamma}$; moreover, this implies that $D_{s}^{-1} \Omega \alpha_{s}$, and consequently $\Omega^{2} \alpha_{s}=\left((1 / 2) D_{s}^{2}+2 \kappa+\kappa_{s} D_{s}^{-1}\right) \Omega \alpha_{s}$ are defined on $S^{1}$. 
We assume that the proposition holds for $m=l$ for some $l \geq 1$. Then, for $X \in T_{\gamma} \mathcal{M}\left(C_{l+1}\right)=$ $T_{\gamma} \mathcal{M}\left(C_{l}\right) \cap \operatorname{Ker}\left(d H_{l+1}\right)_{\gamma}$, by using (10) we get

$$
\begin{aligned}
\int_{S^{1}} \Omega^{l+1} \alpha_{s} d s & =\int_{S^{1}} \kappa \Omega^{l} \alpha_{s} d s=\int_{S^{1}}\left(\left(D_{s}^{-1} \Omega D_{s}\right)^{l} \kappa\right) \cdot \alpha_{s} d s=\int_{S^{1}}\left(D_{s}^{-1} \Omega^{l} \kappa_{s}\right) \cdot \alpha_{s} d s \\
& =\omega_{0}\left(X_{l+1}, X\right)=d H_{l+1}(X)=0,
\end{aligned}
$$

which implies that $\Omega^{l+2} \alpha_{s}$ is determined as a function on $S^{1}$ in the same way as in the case $m=1$.

From Proposition 6 , we can define a tensor field $\omega_{m+1}$ of type $(0,2)$ on $\mathcal{M}\left(C_{m}\right)$ by

$$
\omega_{m+1}(X, Y)=\int_{S^{1}} \alpha \Omega^{m+1} \beta_{s} d s,
$$

which is shown to be skew-symmetric by using (10). Furthermore, in a similar way to the proof of Theorem 2, we see that $\omega_{m+1}$ is a presymplectic form and $X_{n}$ is a Hamiltonian vector field for the Hamiltonian function $H_{n+m+1}$ with respect to $\omega_{m+1}$; indeed, for functions $F$, $G$ given by (7) and for an integer $k$, putting

$$
\{F, G\}_{k}=\int_{S^{1}} \frac{\delta F}{\delta \kappa} \Omega^{k} D_{s} \frac{\delta G}{\delta \kappa} d s
$$

we have a family of Poisson brackets $\{\cdot, \cdot\}_{k}$ with

$$
X_{n}=-\left\{H_{n+2-k}, \cdot\right\}_{k} .
$$

Setting $\widetilde{\alpha}_{F}=D_{s}^{-1} \Omega^{-m} D_{s}(\delta F / \delta \kappa)$ and $\left(\widetilde{X}_{F}\right)_{\gamma}=-(1 / 2)\left(\widetilde{\alpha}_{F}\right)_{s} \gamma+\widetilde{\alpha}_{F} \gamma_{s}$, we have

$$
\omega_{m+1}\left(\widetilde{X}_{F}, X\right)=d F(X) \quad \text { and } \quad \omega_{m+1}\left(\widetilde{X}_{F}, \widetilde{X}_{G}\right)=\{F, G\}_{1-m},
$$

which implies that $\omega_{m+1}$ is presymplectic. Moreover, since

$$
\widetilde{X}_{F} G=-\{F, G\}_{1-m}
$$

holds, we have $X_{n}=\widetilde{X}_{H_{n+m+1}}$ and

$$
\omega_{m+1}\left(X_{n}, \cdot\right)=\omega_{m+1}\left(\widetilde{X}_{H_{n+m+1}}, \cdot\right)=d H_{n+m+1} .
$$

Hence $X_{n}$ is a Hamiltonian vector field of $H_{n+m+1}$ with respect to $\omega_{m+1}$.

Besides $\omega_{m+1}$, we have $m+1$ more presymplectic forms on $\mathcal{M}\left(C_{m}\right)$ by restricting $\omega_{0}, \omega_{1}$ on $\mathcal{M}$ and $\omega_{k+1}$ 's on $\mathcal{M}\left(C_{k}\right)$ 's for $k=1,2, \ldots, m-1$ to $\mathcal{M}\left(C_{m}\right)$; we denote them by the same symbols. By the discussion so far, we obtain the following theorem.

Theorem 7. On $\mathcal{M}\left(C_{m}\right)$, for each $n \in \mathbb{N}$ and $k=0,1, \ldots, m+1, X_{n}$ is a Hamiltonian vector field for $H_{n+k}$ with respect to $\omega_{k}$, that is, the set $\left(\left\{H_{n}\right\}_{n \in \mathbb{N}},\left\{\omega_{k}\right\}_{k=0}^{m+1}\right)$ is a multi-Hamiltonian system on $\mathcal{M}\left(C_{m}\right)$ describing the higher $K d V$ flows.

As on $\mathcal{M}$, we have the following theorem for a Hamiltonian $S^{1}$-action on $\mathcal{M}\left(C_{m}\right)$ :

$$
\mathcal{M}\left(C_{m}\right) \ni \gamma \mapsto \gamma(\cdot+\sigma) \in \mathcal{M}\left(C_{m}\right), \quad \sigma \in S^{1} .
$$

Theorem 8. The moment map $\mu_{m+1}$ for the $S^{1}$-action on $\mathcal{M}\left(C_{m}\right)$ with respect to $\omega_{m+1}$ is given by

$$
\mu_{m+1}(\gamma)\left(\frac{\partial}{\partial \sigma}\right)=H_{m+1}(\gamma), \quad \gamma \in \mathcal{M}\left(C_{m}\right)
$$


Remark 9. We can define $\omega_{m+1}$ in a manner similar to the definitions of $\omega_{0}$ and $\omega_{1}$. We put a map $\phi$ from $T_{\gamma} \mathcal{M}$ to the space of all vector fields along $\gamma$ as

$$
\phi X=-\alpha_{s} \gamma, \quad X=-\frac{1}{2} \alpha_{s} \gamma+\alpha \gamma_{s}
$$

For any tangent vector $X$ of $\mathcal{M},\left(D_{s}^{2}+\kappa\right) X$ has no $\gamma_{s}$-component and it belongs to the image of $\phi$ if $X$ is tangent to $\mathcal{M}\left(C_{1}\right)$. Then for $X \in T_{\gamma} \mathcal{M}\left(C_{1}\right)$ we have

$$
\phi^{-1}\left(D_{s}^{2}+\kappa\right) X=-\frac{1}{2}\left(\Omega \alpha_{s}\right) \gamma+\left(D_{s}^{-1} \Omega \alpha_{s}\right) \gamma_{s}
$$

and

$$
\left(D_{s}^{2}+\kappa\right) \phi^{-1}\left(D_{s}^{2}+\kappa\right) X=-\left(\Omega^{2} \alpha_{s}\right) \gamma
$$

Hence

$$
\int_{S^{1}} \operatorname{det}\left(\begin{array}{c}
X \\
\left(D_{s}^{2}+\kappa\right) \phi^{-1}\left(D_{s}^{2}+\kappa\right) Y
\end{array}\right) d s=\omega_{2}(X, Y)
$$

holds. More generally, $\left[\phi^{-1}\left(D_{s}^{2}+\kappa\right)\right]^{m} X$ can be defined for any tangent vector $X$ of $\mathcal{M}\left(C_{m}\right)$ and we obtain

$$
\int_{S^{1}} \operatorname{det}\left(\begin{array}{c}
X \\
\left(D_{s}^{2}+\kappa\right)\left[\phi^{-1}\left(D_{s}^{2}+\kappa\right)\right]^{m} Y
\end{array}\right) d s=\omega_{m+1}(X, Y)
$$

on $\mathcal{M}\left(C_{m}\right)$. We note that this formula is valid in the case $\omega_{1}(m=0)$ and even in the case $\omega_{0}$ $(m=-1)$ since

$$
\int_{S^{1}} \operatorname{det}\left(\begin{array}{c}
X \\
\phi Y
\end{array}\right) d s=\omega_{0}(X, Y)
$$

\section{A bi-Hamiltonian structure on the space of closed curves in the Euclidean plane}

We denote by $\mathbb{E}^{2}$ the Euclidean plane equipped with the standard inner product $\langle\cdot, \cdot\rangle$, and we set the space $\hat{\mathcal{M}}$ of closed curves in the Euclidean plane $\mathbb{E}^{2}$ by

$$
\hat{\mathcal{M}}=\left\{\hat{\gamma}: S^{1} \rightarrow \mathbb{E}^{2} \mid\left\langle\hat{\gamma}_{s}(s), \hat{\gamma}_{s}(s)\right\rangle \equiv 1\right\}
$$

For $\hat{\gamma} \in \hat{\mathcal{M}}$, the curvature $\hat{\kappa}$ is defined by $T_{s}=\hat{\kappa} N$, where $T=\hat{\gamma}_{s}$ is the velocity vector field and $N$ is the left-oriented unit normal vector field along $\hat{\gamma}$.

Let $\hat{\gamma}(\cdot, t) \in \hat{\mathcal{M}}$ be a one-parameter family of closed curves in $\mathbb{E}^{2}$. Then $\hat{\gamma}_{t}$ is represented as

$$
\hat{\gamma}_{t}=\lambda T+\mu N, \quad \lambda, \mu: S^{1} \rightarrow \mathbb{R}, \quad \lambda_{s}=\hat{\kappa} \mu,
$$

and the curvature $\hat{\kappa}$ evolves as

$$
\hat{\kappa}_{t}=\mu_{s s}+\hat{\kappa} \lambda_{s}+\hat{\kappa}_{s} \lambda=\hat{\Omega}(2 \mu)
$$

where

$$
\hat{\Omega}=\frac{1}{2}\left(D_{s}^{2}+\hat{\kappa}^{2}+\hat{\kappa}_{s} D_{s}^{-1} \hat{\kappa}\right)
$$


is the recursion operator of the $m K d V$ equation:

$$
\hat{\kappa}_{t}=\hat{\Omega} \hat{\kappa}_{s}=\frac{1}{2} \hat{\kappa}_{s s s}+\frac{3}{4} \hat{\kappa}^{2} \hat{\kappa}_{s} .
$$

Hence when we choose $\mu=(1 / 2) \hat{\Omega}^{n-1} \hat{\kappa}_{s}$, we have the $n$th mKdV equation for $\hat{\kappa}$ :

$$
\hat{\kappa}_{t}=\hat{\Omega}^{n} \hat{\kappa}_{s} .
$$

The tangent space of $\hat{\mathcal{M}}$ at $\hat{\gamma} \in \hat{\mathcal{M}}$ is described as

$$
T_{\hat{\gamma}} \hat{\mathcal{M}}=\left\{\lambda T+\mu N \mid \lambda, \mu: S^{1} \rightarrow \mathbb{R}, \lambda_{s}=\hat{\kappa} \mu\right\},
$$

and we can define a presymplectic form $\hat{\omega}_{0}$ on $\hat{\mathcal{M}}$ by

$$
\hat{\omega}_{0}(X, Y)=\int_{S^{1}}\left\langle D_{s} X, Y\right\rangle d s, \quad X, Y \in T_{\hat{\gamma}} \hat{\mathcal{M}}
$$

When $X$ and $Y$ are given by

$$
X=\lambda T+\mu N, \quad Y=\tilde{\lambda} T+\tilde{\mu} N, \quad \lambda, \mu, \tilde{\lambda}, \tilde{\mu}: S^{1} \rightarrow \mathbb{R},
$$

we have

$$
\hat{\omega}_{0}(X, Y)=\int_{S^{1}}\left(\hat{\kappa} \lambda+\mu_{s}\right) \tilde{\mu} d s
$$

and we see that the kernel of $\hat{\omega}_{0}$ at $\hat{\gamma}$ is $\mathbb{R} \cdot \hat{\gamma}_{s}$.

As in the case of the higher $\mathrm{KdV}$ equation (3), the $n$th $\mathrm{mKdV}$ equation (11) can be written as

$$
\hat{\kappa}_{t}=D_{s} \frac{\delta \hat{H}_{n+2}}{\delta \hat{\kappa}}
$$

for an infinite series of conserved quantities $\left\{\hat{H}_{m}\right\}_{m \in \mathbb{N}}$ expressed in the form of

$$
\hat{H}_{m}=\int_{S^{1}} \hat{h}_{m}\left(\hat{\kappa}, \hat{\kappa}_{s}, \hat{\kappa}_{s s}, \ldots\right) d s
$$

where $\hat{h}_{m}$ is a polynomial in $\hat{\kappa}$ and its derivatives up to order $m$, for example,

$$
\hat{h}_{1}=\frac{1}{4} \hat{\kappa}^{2}, \quad \hat{h}_{2}=\frac{1}{32} \hat{\kappa}^{4}-\frac{1}{8} \hat{\kappa}_{s}^{2}, \quad \hat{h}_{3}=\frac{1}{128} \hat{\kappa}^{6}-\frac{5}{32} \hat{\kappa}^{2} \hat{\kappa}_{s}^{2}+\frac{1}{16} \hat{\kappa}_{s s}^{2} .
$$

For each $n \in \mathbb{N}$, we define a vector field $\hat{X}_{n}$ on $\hat{\mathcal{M}}$ by

$$
\left(\hat{X}_{n}\right)_{\hat{\gamma}}=\frac{1}{2}\left(D_{s}^{-1}\left(\hat{\kappa} \hat{\Omega}^{n-1} \hat{\kappa}_{s}\right)\right) T+\frac{1}{2}\left(\hat{\Omega}^{n-1} \hat{\kappa}_{s}\right) N, \quad \hat{\gamma} \in \hat{\mathcal{M}}
$$

then we have the following.

Proposition 10 ([15]). For each $n \in \mathbb{N}, \hat{X}_{n}$ is a Hamiltonian vector field for $\hat{H}_{n}$ with respect to $\hat{\omega}_{0}$. Hence $\hat{H}_{n}$ is a Hamiltonian function for the $n$th $m K d V$ flow $\hat{\gamma}_{t}=\hat{X}_{n}$.

In addition, we define another form $\hat{\omega}_{1}$ on $\hat{\mathcal{M}}$ by

$$
\hat{\omega}_{1}(X, Y)=\int_{S^{1}}\left\langle D_{s} X, D_{s}^{2} Y\right\rangle d s, \quad X, Y \in T_{\hat{\gamma}} \hat{\mathcal{M}},
$$

which is represented as

$$
\hat{\omega}_{1}(X, Y)=\int_{S^{1}}\left(\hat{\kappa} \lambda+\mu_{s}\right) \hat{\Omega} \tilde{\mu} d s
$$

for $X, Y$ given by (12). The following theorem is proved in a similar way to the proof of Theorem 2. 
Theorem 11. The form $\hat{\omega}_{1}$ is a presymplectic form on $\hat{\mathcal{M}}$. For each $n \in \mathbb{N}, \hat{X}_{n}$ is a Hamiltonian vector field for $\hat{H}_{n+1}$ with respect to $\hat{\omega}_{1}$.

Note that the Euclidean motion group $E(2)=O(2) \ltimes \mathbb{R}^{2}$ of $\mathbb{E}^{2}$ acts on $\hat{\mathcal{M}}$. It is easily verified that $\hat{\omega}_{1}$ is invariant under the $E(2)$-action and the kernel of $\hat{\omega}_{1}$ at $T_{\hat{\gamma}} \hat{\mathcal{M}}$ contains the tangent space of the orbit. Hence $\omega_{1}$ determines a presymplectic form on $\hat{\mathcal{M}} / E(2)$.

As well as on $\left(\mathcal{M}, \omega_{1}\right), S^{1}$ acts on $\hat{\mathcal{M}}$ leaving $\hat{\omega}_{1}$ invariant and the following theorem holds.

Theorem 12. The moment map $\hat{\mu}_{1}$ for the $S^{1}$-action on $\hat{\mathcal{M}}$ with respect to $\hat{\omega}_{1}$ is given by

$$
\hat{\mu}_{1}(\hat{\gamma})\left(\frac{\partial}{\partial \sigma}\right)=\hat{H}_{1}(\hat{\gamma}), \quad \hat{\gamma} \in \hat{\mathcal{M}}
$$

\section{The geometric Miura transformation and multi-Hamiltonian structures on spaces of closed curves in the Euclidean plane}

First, we briefly review the geometric Miura transformation which relates the Hamiltonian structures on $\mathcal{M}$ and on $\hat{\mathcal{M}}$ (see [15] for more details). We consider the complexification of $\mathcal{M}$ :

$$
\mathcal{M}^{\mathbb{C}}=\left\{\gamma: S^{1} \rightarrow \mathbb{C}^{2} \backslash\{0\} \mid \operatorname{det}\left(\begin{array}{c}
\gamma \\
\gamma_{s}
\end{array}\right)=1\right\}
$$

We determine the curvature of $\gamma \in \mathcal{M}^{\mathbb{C}}$, (complex) presymplectic forms on $\mathcal{M}^{\mathbb{C}}$, etc. by the same formulas as in the case of $\mathcal{M}$, hence we use the same symbols $\kappa, \omega_{0}, \omega_{1}, \ldots$ to denote them.

By identifying the range $\mathbb{E}^{2}$ of $\hat{\gamma} \in \hat{\mathcal{M}}$ with a complex plane $\mathbb{C}$, we define the geometric Miura transformation $\Phi: \hat{\mathcal{M}} \rightarrow \mathcal{M}^{\mathbb{C}}$ by

$$
\Phi(\hat{\gamma})=\left(-\hat{\gamma}_{s}\right)^{-\frac{1}{2}}(\hat{\gamma}, 1), \quad \hat{\gamma} \in \hat{\mathcal{M}}
$$

The curvature $\kappa$ of $\Phi(\hat{\gamma})$ is related with the curvature $\hat{\kappa}$ of $\hat{\gamma}$ by the Miura transformation:

$$
\kappa=\frac{\sqrt{-1}}{2} \hat{\kappa}_{s}+\frac{1}{4} \hat{\kappa}^{2} .
$$

Moreover, we have the following.

Proposition 13 ([15]). For each $n \in \mathbb{N}, \Phi_{*} \hat{X}_{n}=X_{n}$ holds and the Hamiltonian system $\left(\hat{\omega}_{0}, \hat{H}_{n}\right)$ on $\hat{\mathcal{M}}$ coincides with the pullback of $\left(\omega_{0}, H_{n}\right)$ on $\mathcal{M}^{\mathbb{C}}$ by $\Phi$ :

$$
\hat{\omega}_{0}=\Phi^{*} \omega_{0}, \quad \hat{H}_{n}=\Phi^{*} H_{n} .
$$

For a sequence of real numbers $C=\left\{c_{k}\right\}_{k \in \mathbb{N}}$, the second equation of (14) implies that

$$
\hat{\mathcal{M}}\left(C_{m}\right)=\hat{H}_{1}^{-1}\left(c_{1}\right) \cap \cdots \cap \hat{H}_{m}^{-1}\left(c_{m}\right)=\Phi^{-1}\left(\mathcal{M}^{\mathbb{C}}\left(C_{m}\right)\right) .
$$

Therefore, $\Phi$ gives a map from $\hat{\mathcal{M}}\left(C_{m}\right)$ to $\mathcal{M}^{\mathbb{C}}\left(C_{m}\right)$ and we have a presymplectic form $\hat{\omega}_{m+1}=$ $\Phi^{*} \omega_{m+1}$ on $\hat{\mathcal{M}}\left(C_{m}\right)$. Under these settings the following theorems are directly deduced from Theorems 7 and 8 .

Theorem 14. On $\hat{\mathcal{M}}\left(C_{m}\right)$, for each $n \in \mathbb{N}$ and $k=0,1, \ldots, m+1, \hat{X}_{n}$ is a Hamiltonian vector field for $\hat{H}_{n+k}$ with respect to $\hat{\omega}_{k}$, that is, the set $\left(\left\{\hat{H}_{n}\right\}_{n \in \mathbb{N}},\left\{\hat{\omega}_{k}\right\}_{k=0}^{m+1}\right)$ is a multi-Hamiltonian system on $\hat{\mathcal{M}}\left(C_{m}\right)$ describing the higher modified $K d V$ flows. 
Theorem 15. The moment map $\hat{\mu}_{m+1}$ for the $S^{1}$-action on $\hat{\mathcal{M}}\left(C_{m}\right)$ with respect to $\hat{\omega}_{m+1}$ is given by

$$
\hat{\mu}_{m+1}(\hat{\gamma})\left(\frac{\partial}{\partial \sigma}\right)=\hat{H}_{m+1}(\hat{\gamma}), \quad \hat{\gamma} \in \hat{\mathcal{M}}\left(C_{m}\right)
$$

Remark 16. The symplectic form $\omega_{m+1}$ can be represented as

$$
\hat{\omega}_{m+1}(X, Y)=\int_{S^{1}}\left(\hat{\kappa} \lambda+\mu_{s}\right) \hat{\Omega}^{m+1} \tilde{\mu} d s,
$$

where $X$ and $Y$ are tangent vectors on $\hat{\mathcal{M}}\left(C_{m}\right)$ given by (12). In fact, when $\kappa$ and $\hat{\kappa}$ are related by (13), a direct calculation shows an identity

$$
\left(\sqrt{-1} D_{s}+\hat{\kappa}\right) \hat{\Omega}=\Omega\left(\sqrt{-1} D_{s}+\hat{\kappa}\right) ;
$$

thus we have

$$
\begin{aligned}
\hat{\omega}_{m+1}(X, Y) & =\omega_{m+1}\left(\Phi_{*} X, \Phi_{*} Y\right)=\int_{S^{1}}(\lambda+\sqrt{-1} \mu) \Omega^{m+1}(\tilde{\lambda}+\sqrt{-1} \tilde{\mu})_{s} d s \\
& =\int_{S^{1}}(\lambda+\sqrt{-1} \mu) \Omega^{m+1}\left(\sqrt{-1} D_{s}+\hat{\kappa}\right) \tilde{\mu} d s \\
& =\int_{S^{1}}(\lambda+\sqrt{-1} \mu)\left(\sqrt{-1} D_{s}+\hat{\kappa}\right) \hat{\Omega}^{m+1} \tilde{\mu} d s \\
& =\int_{S^{1}}\left[\left(-\sqrt{-1} D_{s}+\hat{\kappa}\right)(\lambda+\sqrt{-1} \mu)\right] \cdot \hat{\Omega}^{m+1} \tilde{\mu} d s \\
& =\int_{S^{1}}\left(\hat{\kappa} \lambda+\mu_{s}\right) \hat{\Omega}^{m+1} \tilde{\mu} d s .
\end{aligned}
$$

We note that $(15)$ implies $\hat{\omega}_{m+1}$ is a real form, though $\omega_{m+1}$ on $\mathcal{M}^{\mathbb{C}}\left(C_{m}\right)$ is complex.

\section{Acknowledgements}

The authors would like to thank the referees' kind and important comments and advice. The first named author is partly supported by JSPS KAKENHI Grant Number 22540070 and by the Kansai University Grant-in-Aid for progress of research in graduate course, 2013. The second named author is partly supported by JSPS KAKENHI Grant Number 22540107.

\section{References}

[1] Anco S.C., Bi-Hamiltonian operators, integrable flows of curves using moving frames and geometric map equations, J. Phys. A: Math. Gen. 39 (2006), 2043-2072, nlin.SI/0512051.

[2] Anco S.C., Hamiltonian flows of curves in $G / \mathrm{SO}(N)$ and vector soliton equations of $\mathrm{mKdV}$ and sine-Gordon type, SIGMA 2 (2006), 044, 18 pages, nlin.SI/0512046.

[3] Anco S.C., Group-invariant soliton equations and bi-Hamiltonian geometric curve flows in Riemannian symmetric spaces, J. Geom. Phys. 58 (2008), 1-37, nlin.SI/0703041.

[4] Anco S.C., Hamiltonian curve flows in Lie groups $G \subset \mathrm{U}(N)$ and vector NLS, mKdV, sine-Gordon soliton equations, in Symmetries and Overdetermined Systems of Partial Differential Equations, IMA Vol. Math. Appl., Vol. 144, Springer, New York, 2008, 223-250, nlin.SI/0610075.

[5] Anco S.C., Asadi E., Quaternionic soliton equations from Hamiltonian curve flows in $\mathbb{H}^{n}$, J. Phys. A: Math. Theor. 42 (2009), 485201, 25 pages, arXiv:0905.4215.

[6] Anco S.C., Asadi E., Symplectically invariant soliton equations from non-stretching geometric curve flows, J. Phys. A: Math. Theor. 45 (2012), 475207, 37 pages, arXiv:1206.4040. 
[7] Anco S.C., Myrzakulov R., Integrable generalizations of Schrödinger maps and Heisenberg spin models from Hamiltonian flows of curves and surfaces, J. Geom. Phys. 60 (2010), 1576-1603, arXiv:0806.1360.

[8] Anco S.C., Vacaru S.I., Curve flows in Lagrange-Finsler geometry, bi-Hamiltonian structures and solitons, J. Geom. Phys. 59 (2009), 79-103, math-ph/0609070.

[9] Calini A., Ivey T., Marí-Beffa G., Remarks on KdV-type flows on star-shaped curves, Phys. D 238 (2009), 788-797, arXiv:0808.3593.

[10] Chou K.-S., Qu C., The KdV equation and motion of plane curves, J. Phys. Soc. Japan 70 (2001), 19121916.

[11] Chou K.-S., Qu C., Integrable equations arising from motions of plane curves, Phys. D 162 (2002), 9-33.

[12] Chou K.-S., Qu C., Integrable motions of space curves in affine geometry, Chaos Solitons Fractals 14 (2002), $29-44$.

[13] Fujioka A., Kurose T., Motions of curves in the complex hyperbola and the Burgers hierarchy, Osaka J. Math. 45 (2008), 1057-1065.

[14] Fujioka A., Kurose T., Geometry of the space of closed curves in the complex hyperbola, Kyushu J. Math. 63 (2009), 161-165.

[15] Fujioka A., Kurose T., Hamiltonian formalism for the higher KdV flows on the space of closed complex equicentroaffine curves, Int. J. Geom. Methods Mod. Phys. 7 (2010), 165-175.

[16] Hasimoto H., A soliton on a vortex filament, J. Fluid Mech. 51 (1972), 477-485.

[17] Kruskal M.D., Miura R.M., Gardner C.S., Zabusky N.J., Korteweg-de Vries equation and generalizations. V. Uniqueness and nonexistence of polynomial conservation laws, J. Math. Phys. 11 (1970), 952-960.

[18] Kulish P.P., Reiman A.G., Hierarchy of symplectic forms for the Schrödinger equation and for the Dirac equation on a line, J. Sov. Math. 22 (1983), 1627-1637.

[19] Lamb Jr. G.L., Solitons and the motion of helical curves, Phys. Rev. Lett. 37 (1976), 235-237.

[20] Lax P.D., Integrals of nonlinear equations of evolution and solitary waves, Comm. Pure Appl. Math. 21 (1968), 467-490.

[21] Liu Y., Qu C., Zhang Y., Stability of periodic peakons for the modified $\mu$-Camassa-Holm equation, Phys. D 250 (2013), 66-74.

[22] Magri F., A simple model of the integrable Hamiltonian equation, J. Math. Phys. 19 (1978), 1156-1162.

[23] Marí Beffa G., Geometric realizations of bi-Hamiltonian completely integrable systems, SIGMA 4 (2008), 034, 23 pages, arXiv:0803.3866.

[24] Marí Beffa G., Sanders J.A., Wang J.P., Integrable systems in three-dimensional Riemannian geometry, J. Nonlinear Sci. 12 (2002), 143-167.

[25] Miura R.M., Gardner C.S., Kruskal M.D., Korteweg-de Vries equation and generalizations. II. Existence of conservation laws and constants of motion, J. Math. Phys. 9 (1968), 1204-1209.

[26] Newell A.C., Solitons in mathematics and physics, CBMS-NSF Regional Conference Series in Applied Mathematics, Vol. 48, Society for Industrial and Applied Mathematics (SIAM), Philadelphia, PA, 1985.

[27] Olver P.J., Applications of Lie groups to differential equations, Graduate Texts in Mathematics, Vol. 107, Springer-Verlag, New York, 1986.

[28] Pinkall U., Hamiltonian flows on the space of star-shaped curves, Results Math. 27 (1995), 328-332.

[29] Rogers C., Schief W.K., Bäcklund and Darboux transformations. Geometry and modern applications in soliton theory, Cambridge Texts in Applied Mathematics, Cambridge University Press, Cambridge, 2002.

[30] Sanders J.A., Wang J.P., Integrable systems in n-dimensional Riemannian geometry, Mosc. Math. J. 3 (2003), 1369-1393, math.AP/0301212.

[31] Squires S.A., Marí Beffa G., Integrable systems associated to curves in flat Galilean and Minkowski spaces, Appl. Anal. 89 (2010), 571-592.

[32] Terng C.-L., Thorbergsson G., Completely integrable curve flows on adjoint orbits, Results Math. 40 (2001), 286-309, math.DG/0108154. 